\title{
Photoprotective properties of $20(S)$-protopanaxatriol, an aglycone of ginseng saponins: Protection from ultraviolet-B radiation-induced oxidative stress in human epidermal keratinocytes
}

\author{
SUN-JOO OH ${ }^{1}$, KYUNGHOON KIM $^{1}$ and CHANG-JIN LIM ${ }^{2}$ \\ Departments of ${ }^{1}$ Biological Sciences and ${ }^{2}$ Biochemistry, College of Natural Sciences, Kangwon National University, \\ Chuncheon, Gangwon 200-701, Republic of Korea
}

Received July 27, 2015; Accepted June 28, 2016

DOI: $10.3892 / \mathrm{mmr} .2016 .5581$

\begin{abstract}
Ginsenosides are responsible for diverse pharmacological properties ascribed to ginseng, a plant used in traditional medicine. Ginsenosides are classified into three categories: Protopanaxadiol, protopanaxatriol (PPT) and oleanolic acid. As an aglycone of PPT-type ginsenosides, PPT exists in two stereoisomeric forms, 20(S)-PPT and 20(R)-PPT. The 20(S)-PPT stereoisomer is a major metabolic product of PPT-type ginsenosides produced in the gastrointestinal tract. In the present study, 20(S)-PPT suppressed the elevation of reactive oxygen species in HaCaT cells following irradiation with ultraviolet (UV)-B. In addition, 20(S)-PPT inhibited UV-B-induced gelatinase activities of matrix metalloproteinase-2 and -9 in $\mathrm{HaCaT}$ cells, and suppressed UV-B-induced expression and secretion of these proteins. Accordingly, 20(S)-PPT restored the total glutathione levels in UV-B-irradiated keratinocytes. Taken together, these data indicated that 20(S)-PPT may possess photoprotective properties that combat the effects of UV-B radiation.
\end{abstract}

\section{Introduction}

Ginseng, derived from the root of Panax ginseng C.A. Meyer (Araliaceae), has been used as a traditional medicine in Asian

Correspondence to: Professor Kyunghoon Kim, Department of Biological Sciences, College of Natural Sciences, Kangwon National University, 192-1 Hyoja-2-dong, Chuncheon, Gangwon 200-701, Republic of Korea

E-mail:kkim@kangwon.ac.kr

Professor Chang-Jin Lim, Department of Biochemistry, College of Natural Sciences, Kangwon National University, 192-1 Hyoja-2-dong, Chuncheon, Gangwon 200-701, Republic of Korea

E-mail: cjlim@kangwon.ac.kr

Key words: glutathione, matrix metalloproteinase, oxidative stress, $20(S)$-protopanaxatriol, reactive oxygen species, skin anti-photoaging, UV-B radiation countries for centuries. Today it is used as a dietary supplement worldwide. Due to widespread use, documenting pharmacological efficacies has become an important goal. Ginsenosides are believed to be responsible for a broad range of pharmacological properties ascribed to ginseng, including antitumor, anti-inflammatory, antinociceptive, antiallergic and antidiabetic activities (1-7). Ginsenosides, which belong to a class of steroidal glycosides, are composed of an aglycone (sapogenin), including protopanaxadiol, protopanaxatriol (PPT) or oleanolic acid, and sugar moieties attached to the C-3 and C-20 positions. Protopanaxadiol and PPT exist in $S$ - and $R$-stereoisomeric forms, based upon the configuration of $\mathrm{C}-20$. Of the two stereoisomeric forms, 20(S)-PPT (Fig. 1) is the major metabolic product of PPT-type ginsenosides, including Re, Rf, Rg1, Rg2 and Rh1, which are deglycosylated by human intestinal bacteria following oral consumption (8).

Purification of PPT and its stereoisomers has enabled the pharmacological assessment of activities. PPT suppresses the increase in lipopolysaccharide-induced inducible nitric oxide synthase and cyclooxygenase- 2 expression, which occur via inactivation of nuclear factor- $\kappa \mathrm{B}$ by preventing $\mathrm{I}-\kappa \mathrm{B} \alpha$ phosphorylation and degradation (9). This result is not consistent with previous findings indicating that Panax ginseng extract rich in PPT promotes nitric oxide production and relaxation of the vascular endothelium via multiple signaling pathways (10). Peroxisome proliferator-activated receptor (PPAR) $\gamma$, a member of the nuclear receptors of ligand-activated transcription factor family, regulates the expression of genes crucial for lipid and glucose metabolism, and adipose cell differentiation. Accordingly, ligands of PPAR $\gamma$ are potent insulin sensitizers used in the treatment of type 2 diabetes (11). PPT enhances PPAR $\gamma$-transactivation activity and adipogenesis by increasing the expression of PPAR $\gamma$ target genes, including adipocyte fatty acid-binding protein, lipoprotein lipase, phosphoenolpyruvate carboxykinase and glucose transporter 4, in 3T3-L1 adipocytes (11). This finding suggested that PPT may be a PPAR $\gamma$ agonist that can improve insulin resistance associated with diabetes (11).

PPT inhibits corticosteroid production in adrenal fasciculate cells in vivo and inhibits the conversion of cholesterol to pregnenolone (12). Pretreatment of human umbilical vein 
endothelial cells with PPT produces a myriad of effects, including protecting cells against hydrogen peroxide-induced cell injury and death, diminishing hydrogen peroxide-induced DNA damage, and enhancing activation of poly-ADP ribose polymerase and depleting its intracellular substrate $\mathrm{NAD}^{+}$. PPT also prevents decreases in the ratio of glutathione (GSH) to glutathione disulfide caused by hydrogen peroxide, implying that PPT can protect human umbilical vein endothelial cells against hydrogen peroxide-induced cell death by protecting against oxidative stress (13). The 20(S)-PPT stereoisomer also provides protection against myocardial ischemic injury by enhancing the anti-free radical actions of heart tissues (14).

Findings indicated that 20(S)-PPT has antioxidant activities in several cell types. However, photoprotective properties in dermal keratinocytes remain to be elucidated. Reactive oxygen species (ROS), produced by abnormal metabolic reactions and exogenous stress-inducers, including ultraviolet (UV) radiation, causes damage to macromolecules, which can result in physiological dysfunction, genetic mutation, cell aging and cell death (15). UV-B radiation, with wavelengths between 280 and $315 \mathrm{~nm}$, is one well-known cause of skin photoaging, including wrinkle formation, laxity, mottled pigmentation, coarseness, degradation of matrix macromolecules, epidermal thickening, vascularization and immunosuppression $(16,17)$. Keratinocytes, the major cell population in the basal layer of the skin, are the primary targets of UV-B radiation. The present study was performed to determine whether $20(S)$-PPT possesses photoprotective properties that combat the effects of UV-B irradiation in skin.

\section{Materials and methods}

Cell culture. Immortalized human $\mathrm{HaCaT}$ dermal keratinocytes (American Type Culture Collection, Manassas, VA, USA) were grown in Dulbecco's modified Eagle's medium (DMEM; HyClone Laboratories, Logan, UT, USA) containing $10 \%$ heat-inactivated fetal bovine serum (FBS; HyClone Laboratories), $100 \mathrm{U} / \mathrm{ml}$ penicillin and $100 \mu \mathrm{g} / \mathrm{ml}$ streptomycin (HyClone Laboratories) in a humidified atmosphere with $5 \% \mathrm{CO}_{2}$ at $37^{\circ} \mathrm{C}$. Prior to treatments, $1 \times 10^{5} \mathrm{HaCaT}$ cells were seeded into 24 -well plates, cultured overnight, washed twice with $1 \mathrm{ml}$ phosphate-buffered saline (PBS) and replaced with $1 \mathrm{ml} \mathrm{FBS-free} \mathrm{medium.}$

$U V-B$ irradiation. An ultraviolet lamp (peak, $312 \mathrm{~nm}$; VL-6 M; Vilber Lourmat, Marne La Vallée, France) was used as a UV-B source. A radiometer (VLX-3 W; Vilber Lourmat) with a sensor (bandwidth, 280-320 nm; CX-312; Vilber Lourmat) was used to monitor radiation intensity. Mammalian cells were irradiated with UV-B radiation at $70 \mathrm{~mJ} / \mathrm{cm}^{2}$.

Preparation of cellular lysates. Adherent cells were washed twice with PBS and stored on ice for $5 \mathrm{~min}$. The cells were harvested using a cell scraper and centrifuged at $4^{\circ} \mathrm{C}$ for $10 \mathrm{~min}$ at $3,000 \mathrm{x} \mathrm{g}$. The cell pellets were resuspended in cell lysis buffer [50 mM HEPES (pH 7.5), 10\% sucrose and $0.1 \%$ Triton X-100] and were incubated for $30 \mathrm{~min}$ on ice. Cellular lysates were obtained following centrifugation at $3,000 \mathrm{x} \mathrm{g}$ for $15 \mathrm{~min}$ and protein concentrations were determined using the Bradford procedure (18). Bovine serum albumin (BSA; Sigma-Aldrich, St Louis, MO, USA) was used as a standard.

Determination of intracellular ROS. To determine intracellular ROS concentrations in cultured keratinocytes, a redox-sensitive fluorescent probe, $2^{\prime}, 7^{\prime}$-dichlorofluorescein diacetate (DCFH-DA), which produces 2',7'-dichlorofluorescein (DCF; $\lambda_{\text {excitation }}=485 \mathrm{~nm}, \lambda_{\text {emission }}=530 \mathrm{~nm}$ ) was used as previously described (19). Following treatment with 20(S)-PPT $(0,5,12$ and $30 \mu \mathrm{M}$; Ambo Institute, Seoul, Korea) and $20 \mu \mathrm{M}$ DCFH-DA (Sigma-Aldrich) for $30 \mathrm{~min}$ at $37^{\circ} \mathrm{C}$, the cells were washed twice with $1 \mathrm{ml}$ FBS-free medium. The cells were resuspended in $1 \mathrm{ml}$ FBS-free medium and irradiated with $70 \mathrm{~mJ} / \mathrm{cm}^{2}$ UV-B. Intracellular ROS levels were immediately quantified using a Multi-Mode Microplate Reader (Synergy Mx; BioTek Instruments, Winooski, VT, USA). For imaging analyses, the cells were treated with 20(S)-PPT and $20 \mu \mathrm{M}$ dihydrorhodamine-123 (DHR-123; Sigma-Aldrich) for $30 \mathrm{~min}$ at $37^{\circ} \mathrm{C}$, irradiated with $70 \mathrm{~mJ} / \mathrm{cm}^{2} \mathrm{UV}-\mathrm{B}$, and immediately analyzed using confocal laser scanning microscopy (Fluoview-FV300; Olympus, Tokyo, Japan). The assays were repeated at least three times.

Quantitation of nitrite. Accumulated nitrite $\left(\mathrm{NO}_{2}^{-}\right)$in culture supernatants was quantitated using a spectrophotometric assay, based upon the Griess reaction (20). Briefly, an equal volume of Griess reagent ( $1 \%$ sulfanilamide- $0.1 \%$ $\mathrm{N}$-1-naphthyl-ethylenediamine dihydrochloride in $2.5 \%$ phosphoric acid; Sigma-Aldrich) was incubated with culture supernatants for $10 \mathrm{~min}$ at room temperature and the absorbance at $550 \mathrm{~nm}$ was measured using an enzyme-linked immunosorbent assay reader (Molecular Devices, Sunnyvale, CA, USA). A calibration curve was constructed using known concentrations (0-160 $\mu \mathrm{M})$ of sodium nitrite (Sigma-Aldrich).

Cell viability assay. To determine the survival of keratinocytes in the presence of 20(S)-PPT, an MTT assay, which detects metabolic activity (21), was used. The cells were treated with 20(S)-PPT $(0,5,12$ and $30 \mu \mathrm{M})$ for $30 \mathrm{~min}$. After removing the medium, the cells were treated with $5 \mu \mathrm{g} / \mathrm{ml} 3$-(4,5-dimethylthiazol-2-yl)-2,5-diphenyltetrazolium bromide (MTT; Sigma-Aldrich) in the medium. After incubating the cells at $37^{\circ} \mathrm{C}$ for $4 \mathrm{~h}$, the MTT formazan crystals were dissolved in dimethyl sulfoxide (150 $\mu \mathrm{l}$; Sigma-Aldrich). Formazan, generated from reduction of MTT in the mitochondria of living cells, was quantified using the absorbance at $540 \mathrm{~nm}$.

Gelatin zymography. Gelatinase activities of matrix metalloproteinase (MMP)-2 and -9 were determined using zymographic analyses, as previously described (22). The cells were incubated for $24 \mathrm{~h}$ at $37^{\circ} \mathrm{C}$ and were washed twice with $1 \mathrm{ml}$ PBS. Cells, in $1 \mathrm{ml}$ FBS-free medium, were treated with $20(S)$-PPT for $30 \mathrm{~min}$ and irradiated with $70 \mathrm{~mJ} / \mathrm{cm}^{2}$ UV-B. Culture supernatants, obtained from the irradiated culture incubated for $24 \mathrm{~h}$ at $37^{\circ} \mathrm{C}$, were fractionated on $10 \%(\mathrm{w} / \mathrm{v})$ sodium dodecyl sulfate-polyacrylamide gel electrophoresis (SDS-PAGE) gels impregnated with $1 \mathrm{mg} / \mathrm{ml}$ gelatin (Sigma-Aldrich) under non-reducing 


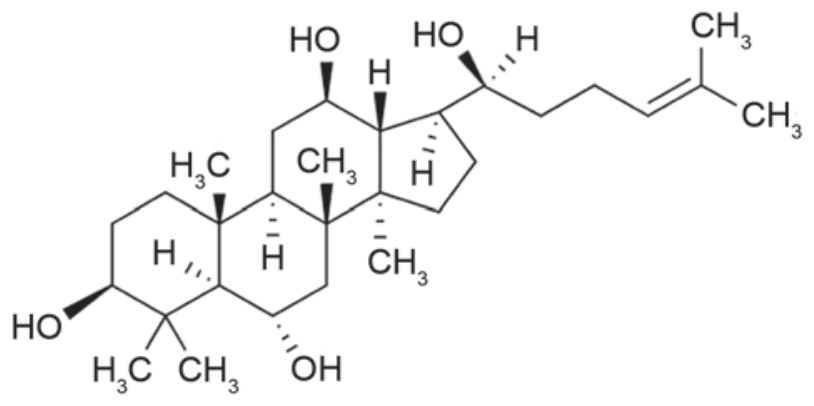

Figure 1. Chemical structure of 20(S)-protopanaxatriol.

conditions. The proteins in the gel were renatured by agitating twice in $2.5 \%$ Triton $\mathrm{X}-100$ at room temperature for $30 \mathrm{~min}$, and incubating in buffer [50 mM Tris buffer ( $\mathrm{pH} 7.8), 5 \mathrm{mM}$ $\mathrm{CaCl}_{2}, 0.15 \mathrm{M} \mathrm{NaCl}$ and $1 \%$ Triton X-100] for $24 \mathrm{~h}$. Following this, the gel was stained with $0.1 \%$ Coomassie Brilliant Blue R-250 and gelatin-degrading enzyme activities were shown as clear zones against the blue background. MMP-2 and MMP-9 activity bands were identified in accordance with molecular mass, which was estimated using molecular mass markers (Quantity One Software, version 4.6.9, Bio-Rad Laboratories, Richmond, CA, USA).

Quantitation of total GSH. As previously described (23), the total GSH content was determined using an enzymatic recycling assay based on glutathione reductase (Sigma-Aldrich). The reaction mixture $(200 \mu \mathrm{l})$, containing $175 \mathrm{mM} \mathrm{KH}_{2} \mathrm{PO}_{4}, 6.3 \mathrm{mM}$ EDTA, $0.21 \mathrm{mM}$ NADPH (Sigma-Aldrich), $0.6 \mathrm{mM}$ DTNB (Sigma-Aldrich) and 0.5 units/ml glutathione reductase, and the cellular lysate was incubated at $25^{\circ} \mathrm{C}$ for $5 \mathrm{~min}$. The changes in absorbance at $412 \mathrm{~nm}$ were monitored using a microplate reader. The total GSH was reported as $\mu \mathrm{g} / \mathrm{mg}$ protein.

Western blotting analysis. To detect the protein expression levels of MMP-2 and MMP-9 in conditioned media and cellular lysates, western blotting was performed. Conditioned media and cellular lysates were separated on $10 \%$ SDS-PAGE gels and electrotransferred onto polyvinylidene membranes. The membranes were blocked at room temperature for $2 \mathrm{~h}$ with blocking buffer (2\% BSA in 1X Tris-buffered saline with $0.1 \%$ Tween-20). Following blocking, the membranes were probed with primary antibody overnight at $4^{\circ} \mathrm{C}$. The following antibodies, after 1,000-fold dilution, were used: Anti-MMP-2 (cat. no. ALX-210-753; Enzo Life Sciences, Farmingdale, NY, USA), anti-MMP-9 (cat. no. \#3852S; Cell Signaling Technology, Inc., Danvers, MA, USA) and anti-glyceraldehyde 3-phosphate dehydrogenase (GAPDH; cat. no. LF-PA0212; Young In Frontier Co., Ltd., Seoul, Korea). After the membranes were washed with PBS, they were subsequently incubated with goat anti-rabbit immunoglobulin G-pAb-horseradish peroxidase-conjugated secondary antibody (cat. no. ADI-SAB-300; Enzo Life Sciences) for $1 \mathrm{~h}$ at room temperature, and were developed using enhanced West-save up ${ }^{\mathrm{TM}}$ (AbFrontier, Seoul, Korea). The band strength, expressed as a percentage of the control, was determined by densitometry using ImageJ software (version 1.48; National Institutes of Health, Bethesda, MA, USA).
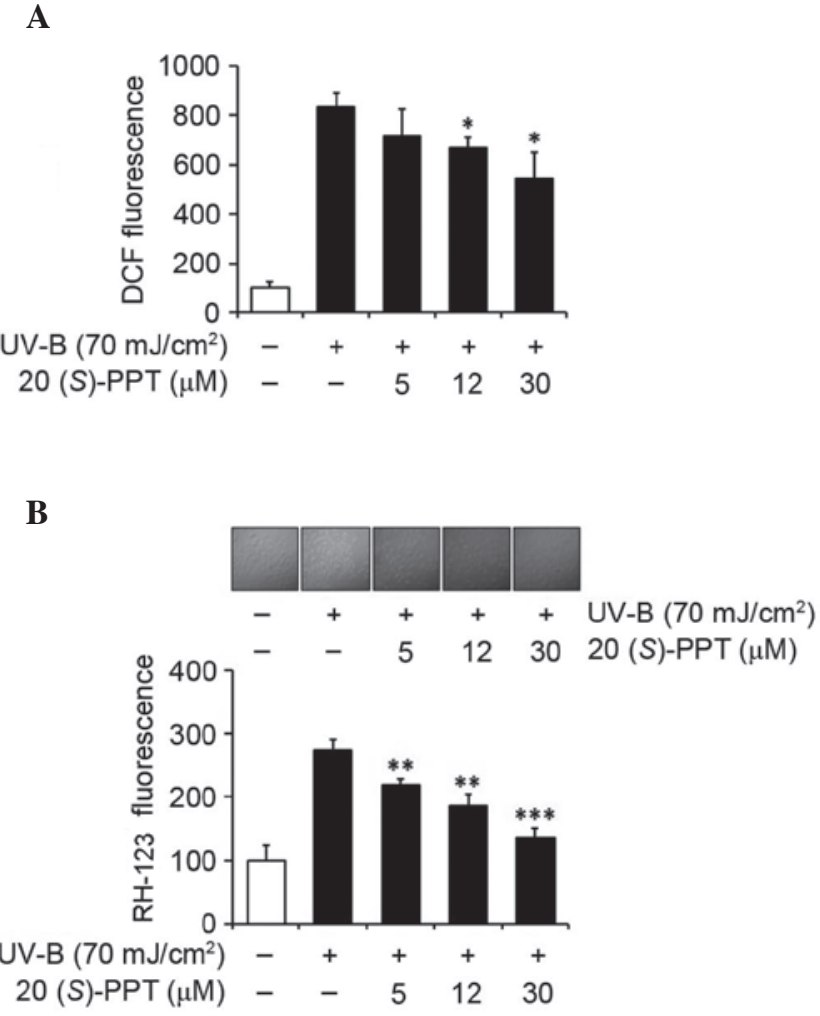

Figure 2. Effects of 20(S)-PPT on the production of ROS. ROS production was detected using (A) fluorescence and (B) confocal microscopy. (A) The levels of ROS are presented as DCF fluorescence arbitrary units, expressed as a percentage of the control. (B) The ROS-associated fluorescent signals, shown by RH-123 fluorescence and expressed as a percentage of the control, were quantified using Adobe Photoshop software. Representative images of three independent experiments are shown. The data are expressed as the mean \pm standard deviation $\left[{ }^{*} \mathrm{P}<0.05 ;{ }^{* *} \mathrm{P}<0.01 ;{ }^{* * *} \mathrm{P}<0.001\right.$ vs. non-treated control (UV-B irradiation alone)]. PPT, protopanaxatriol; UV, ultraviolet; ROS, reactive oxygen species; DCF, dichlorofluorescein; RH-123, rhodamine-123.

Statistical analyses. The data are presented as the mean \pm standard deviation. Comparisons between experimental groups were statistically analyzed using an unpaired Student's t-test. $\mathrm{P}<0.05$ was considered to indicate a statistically significant difference.

\section{Results}

$U V$-B-induced ROS elevation. To determine the effects of $\mathrm{UV}-\mathrm{B}$ radiation on the production of ROS, cultured $\mathrm{HaCaT}$ cells were exposed to $70 \mathrm{~mJ} / \mathrm{cm}^{2} \mathrm{UV}-\mathrm{B}$ radiation. Without pretreatment with $20(S)$-PPT, intracellular ROS levels increased 8.3-fold compared with the non-irradiated cells (Fig. 2A). Pretreatment of cells with 20(S)-PPT attenuated UV-B-induced ROS elevation in a concentration-dependent manner (Fig. 2A). Treatment with 5, 12 and $30 \mu \mathrm{M} 20(S)$-PPT reduced ROS elevation to $85.6,80.2$ and $65.4 \%$, respectively, of irradiated cells without 20(S)-PPT pretreatment (Fig. 2A). The activity of 20(S)-PPT on the UV-B-induced ROS elevation in keratinocytes was also determined by confocal microscopy analyses using dihydrorhodamine-123 (Fig. 2B). Treating cultured HaCaT cells with 5, 12 and $30 \mu \mathrm{M} 20(S)$-PPT prior to UV-B irradiation reduced ROS levels to 79.7, 67.6 and 50.0\%, respectively, of the cultured $\mathrm{HaCaT}$ cells without 20(S)-PPT 
A

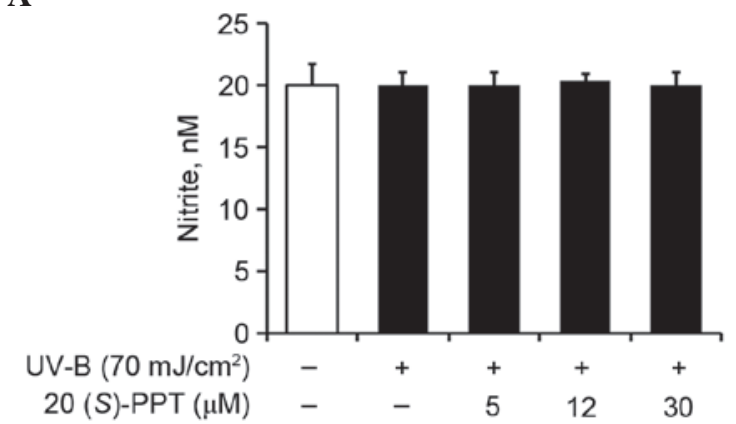

B

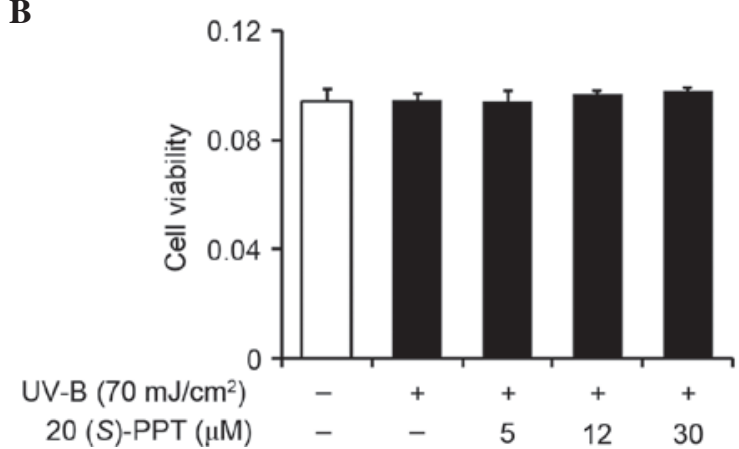

Figure 3. Effects of 20(S)-PPT on nitric oxide levels and cell viability. (A) The levels of nitrite were measured spectrophotometrically following exposure to UV-B or 20(S)-PPT. (B) Cell viability was measured using an 3-(4,5-dimethylthiazol-2-yl)-2,5 diphenyl tetrazolium bromide assay. The data are presented as the mean \pm standard deviation. PPT, protopanaxatriol; $\mathrm{UV}$, ultraviolet.

pretreatment (Fig. 2B). These data indicated that 20(S)-PPT diminishes ROS elevation in keratinocytes following UV-B irradiation.

Nitric oxide production and cell survival. Nitric oxide, synthesized in minute quantities by constitutive nitric oxide synthases, is involved in normal physiological processes. By contrast, it exhibits pathological effects when synthesized in excessive quantities by inducible nitric oxide synthases, which respond to proinflammatory agents. Nitric oxide levels remained unchanged in UV-B-irradiated $\mathrm{HaCaT}$ cells compared with the non-irradiated cells (Fig. 3A). These results were obtained regardless of pretreatment with 20(S)-PPT (Fig. 3A), indicating that 20(S)-PPT fails to modulate nitric oxide levels in UV-B-irradiated $\mathrm{HaCaT}$ cells.

To determine whether UV-B radiation produced cytotoxic affects in HaCaT cells, MTT assays were used to assess cell viability. As shown in Fig. 3B, UV-B irradiation alone did not affect viability. Similarly, pretreatment with 20(S)-PPT caused no alteration of the effects of UV-B irradiation (Fig. 3B), indicating that 20(S)-PPT treatment had no affect on the viability of irradiated keratinocytes.

Activity and expression of MMP-2 and MMP-9. UV irradiation is capable of inducing the expression of certain MMP family members, including MMP-9, MMP-2 and MMP-1, which degrade collagen and other extracellular matrix proteins of the dermal connective tissue. Therefore, chronic exposure to UV radiation alters the architecture of the skin, leading to photoaging (24). ROS scavenging, and suppression of MMP activity
A

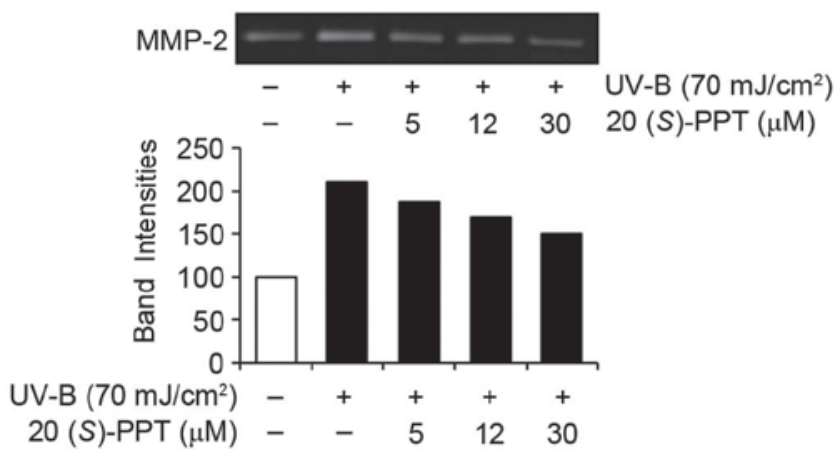

B
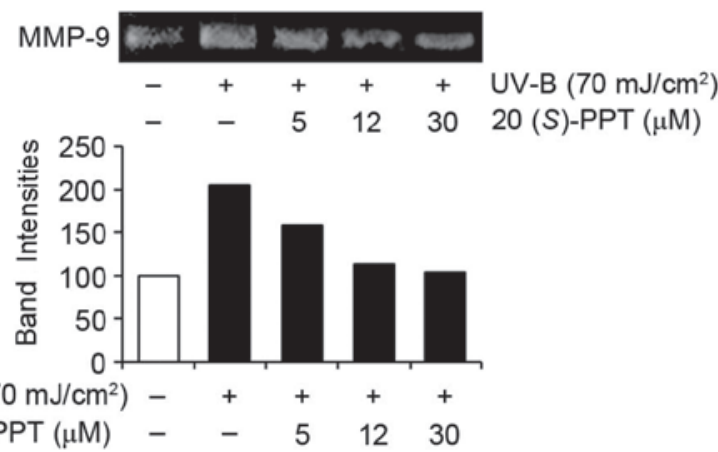

Figure 4. Effects of 20(S)-PPT on the gelatinase activities of MMP-2 and MMP-9. The gelatinase activities of (A) MMP-2 and (B) MMP-9 were determined using gelatin zymography. The relative band intensities are shown and were determined by densitometry using ImageJ software. Representative images from three independent experiments are shown. The data was quantified to show the band intensities. MMP, matrix metalloproteinases; PPT, protopanaxatriol; UV, ultraviolet.

and expression attenuated premature photoaging of skin. As shown in Fig. 4A, 20(S)-PPT attenuated the gelatinase activity of MMP-2, which was significantly enhanced by UV-B irradiation in $\mathrm{HaCaT}$ cells. In a similar manner, the gelatinase activity of MMP-9 was markedly enhanced by UV-B irradiation in HaCaT cells and enhanced MMP-9 activity was diminished by 20(S)-PPT in a concentration-dependent manner (Fig. 4B). These results indicated that 20(S)-PPT attenuates the gelatinase activities of MMP-2 and MMP-9 in keratinocytes, which were enhanced by UV-B irradiation.

The effects of 20(S)-PPT on MMP-2 and MMP-9 were also determined by western blotting analyses of conditioned media and cell lysates (Figs. 5 and 6). UV-B radiation enhanced the protein expression of MMP-2 in conditioned media from $\mathrm{HaCaT}$ cells (Fig. 5A), and 20(S)-PPT reduced UV-B-induced MMP-2 protein levels in conditioned media in a concentration-dependent manner (Fig. 5A). Analyses of cell lysates produced similar results. As shown in Fig. 5B, MMP-2 protein levels in cellular lysates were enhanced by UV-B irradiation and the UV-B-enhanced MMP-2 protein levels were reduced by 20(S)-PPT in a concentration-dependent manner. MMP-9 protein levels in conditioned media were significantly elevated by UV-B irradiation and UV-B-induced MMP-9 protein levels were diminished by 20(S)-PPT (Fig. 6A). Additionally, as shown in Fig. 6B, MMP-9 protein levels in $\mathrm{HaCaT}$ cell lysates were induced by UV-B irradiation and increased MMP-9 protein levels were 
A
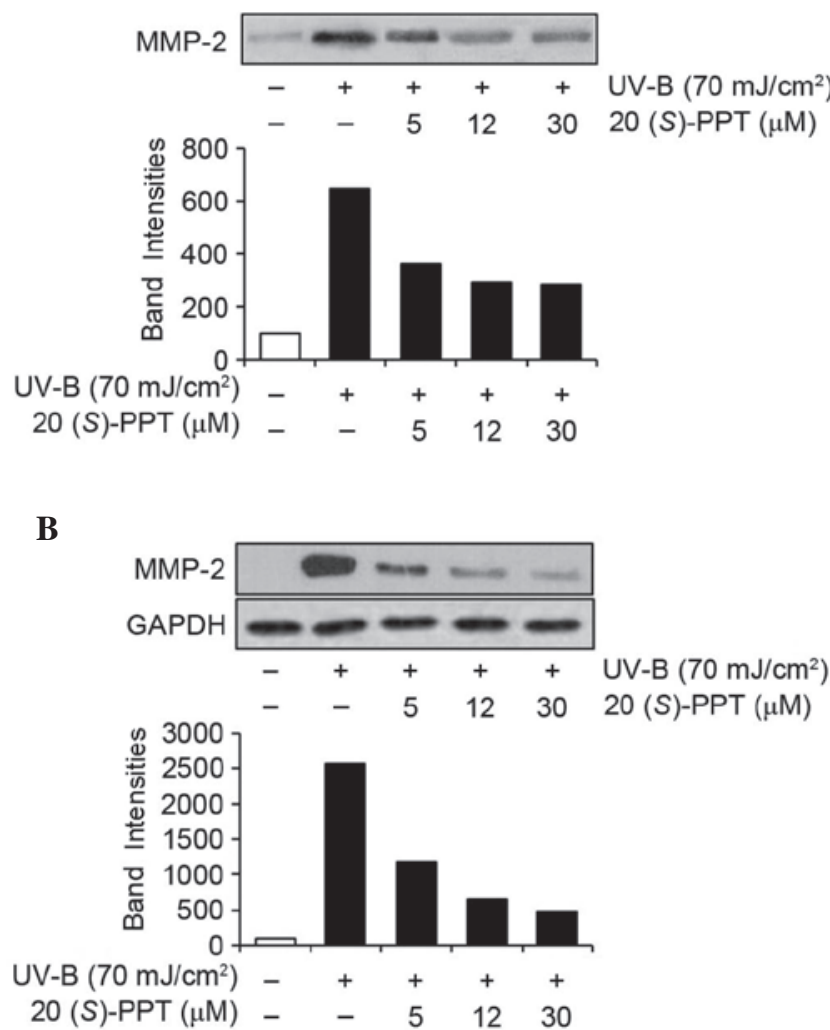

Figure 5. Effects of 20(S)-PPT on MMP-2 protein levels. The protein expression levels of MMP-2 were determined in (A) conditioned media and (B) cellular lysates. GAPDH was used as a loading control. The relative band intensities were determined by densitometry using ImageJ software. Representative images from three independent experiments are shown. PPT, protopanaxatriol; UV, ultraviolet; GAPDH, glyceraldehyde 3-phosphate dehydrogenase.

attenuated by 20(S)-PPT. Collectively, these results indicated that 20(S)-PPT downregulates the UV-B-mediated induction of MMP-2 and MMP-9 in HaCaT cells.

GSH content. The total GSH levels were significantly diminished in $\mathrm{HaCaT}$ cells irradiated with $70 \mathrm{~mJ} / \mathrm{cm}^{2}$ UV-B (Fig. 7). Pretreatment with 5, 12 and $30 \mu \mathrm{M}$ 20(S)-PPT increased GSH levels by 1.7-, 2.5- and 3.3-fold, respectively, compared with irradiated keratinocytes that did not receive pretreatment (Fig. 7). These data indicated that the GSH-enhancing effects of 20(S)-PPT assisted with combating the effects of UV-B irradiation.

\section{Discussion}

The present study revealed that 20(S)-PPT, a major metabolic intermediate of PPT-type ginsenosides, diminished ROS levels in keratinocytes, suppressed the gelatinase activities of MMP-2 and MMP-9, and decreased the protein expression levels of MMP-2 and MMP-9 in cellular lysates and media. These data suggested that 20(S)-PPT and associated PPT-type ginsenosides, including Re, Rf, Rg1, Rg2 and Rh1, may have antiphotoaging properties.

Oxidative stress, caused by imbalanced pro-oxidant processes and antioxidant defense systems, occurs when
A

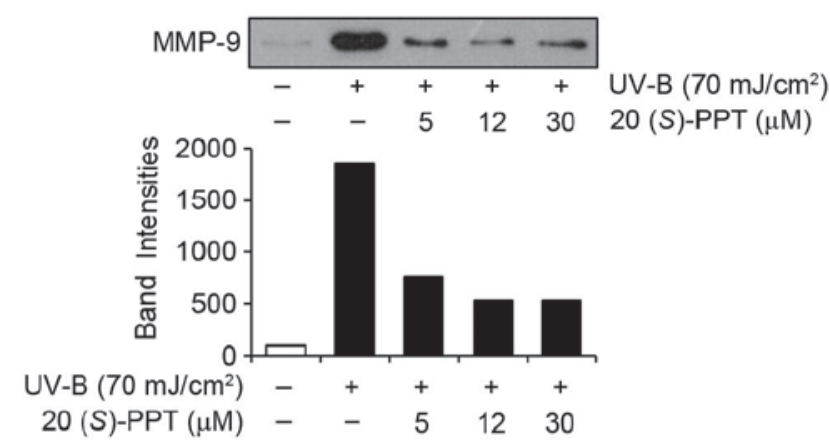

B

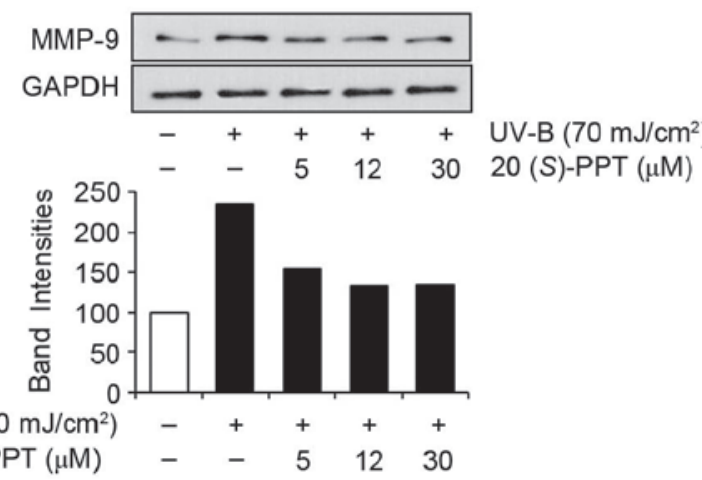

Figure 6. Effects of 20(S)-PPT on MMP-9 protein levels. The protein expression levels of MMP-9 were determined in (A) conditioned media and (B) cellular lysates. GAPDH was used as a loading control. The relative band intensities was determined by densitometry using ImageJ software. Representative images from three independent experiments are shown. PPT, protopanaxatriol; UV, ultraviolet; GAPDH, glyceraldehyde 3-phosphate dehydrogenase.

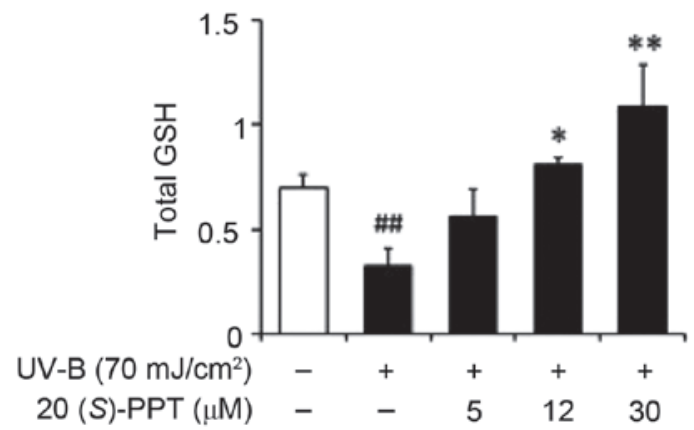

Figure 7. Effects of 20(S)-PPT on the total levels of GSH. The total levels of GSH ( $\mu \mathrm{g} / \mathrm{mg}$ protein) were determined using an enzymatic recycling assay. The data are expressed as the mean \pm standard deviation $\left[{ }^{\# \#} \mathrm{P}<0.01\right.$ vs. non-irradiated control; ${ }^{*} \mathrm{P}<0.05$ and ${ }^{* *} \mathrm{P}<0.01$ vs. non-treated control (UV-B irradiation alone)]. GSH, glutathione; PPT, protopanaxatriol; UV, ultraviolet.

cellular redox homeostasis systems are disturbed. Chronic exposure to UV light from the sun or artificial sources causes oxidative stress, which results in the photoaging of skin. UV-A and UV-B radiation from sunlight is hypothesized to be responsible for $\sim 90 \%$ of skin photoaging $(25,26)$. UV-B radiation increases the production of ROS, which cause oxidative damage in epidermal and dermal cells, and severe oxidative stress may cause apoptotic or necrotic death in dermal cells (16). UV radiation also induces 
the expression and secretion of MMP-1, MMP-2, MMP-3, MMP-9 and MMP-13 in the epidermis and dermis, which contributes to skin damage and photoaging $(27,28)$.

Collagen destruction is closely associated with the induction of MMPs, which are secreted by epidermal keratinocytes and dermal fibroblasts (29). Since skin photoaging is also a leading cause of skin cancer, inhibiting the elevation of ROS and MMP activity may reduce skin photoaging and cancer risk. Several crude plant extracts that protect skin by downregulating ROS and/or MMPs have been previously identified (30-33). A glutathione peroxidase mimic, 2-selenium-bridged $\beta$-cyclodextrin, reverses apoptosis and lipid peroxidation in keratinocytes (34).

GSH is a well-known small-molecule antioxidant with central roles in maintaining cellular redox homeostasis and protecting against oxidative stress and injury. UV-B irradiation-induced GSH depletion in cultured human keratinocytes mimics the pathogenesis of several cutaneous disorders. Decreased activity of $\gamma$-glutamylcysteine synthetase, a rate-limiting enzyme in GSH biosynthesis, and diminished cysteine uptake through the functional inhibition of the system Xc(-), a cysteine transporter on the cell membrane (35), are implicated in the pathogenesis. Indeed, inactivation of the cysteine transporter system is a major contributor to the UV-B-induced decrease of GSH levels in human keratinocytes (35). GSH depletion has also been shown in a commercially available reconstituted human epidermis model of UV-B radiation (36). When mouse lenses are exposed to UV-B radiation, simultaneous decreases in ATP and GSH, which can be prevented by caffeine, were observed (37). In the aquatic organism Tubifex, UV-B radiation induced the production of singlet oxygen, superoxide anions and hydroxyl radicals, and diminished the levels of GSH, DNA, RNA and protein (38).

Several pure ginsenosides can restore GSH levels after depletion caused by stress. Ginsenoside Rg1 decreased malondialdehyde levels and intracellular ROS, and enhanced superoxide dismutase activity and the total GSH in colistin-treated rat pheochromocytoma cells, implying that neuroprotective effects may be mediated via the inhibition of oxidative stress (39). Similarly, Rg1 enhanced the total GSH in Schwann cells exposed to hydrogen peroxide (40), and $\mathrm{Rb} 2$, a protopanaxadiol-type ginsenoside, decreased blood malondialdehyde and elevated total GSH in ovariectomized mice. These data suggested that the anti-osteoporosis effects of $\mathrm{Rb} 2$ may be linked to reduced oxidative damage (41). Another ginsenoside, Re, also restores GSH levels in the serum of diabetic rats and attenuates diabetes-associated cognitive decline (42). Ginsenoside Rd was shown to increase the total GSH and promote the antioxidant activities of catalase, superoxide dismutase and glutathione peroxidase in cultured hippocampal neurons exposed to oxygen-glucose deprivation (43); Rd also enhanced total GSH and levels of $\gamma$-glutamylcysteine synthetase heavy chain in a rat hepatocyte cell line (44). Collectively, although GSH-restoring effects were not detected in the previous experiments using UV-B irradiation, certain ginsenosides were shown to enhance GSH levels that had declined under other types of conditions (42-44).
The present results confirmed that 20(S)-PPT suppresses the GSH depletion caused by UV-B exposure. Although the mechanisms of these protective activities remain unknown, restoration of GSH levels may be an initiating event that leads to lowering of ROS in keratinocytes.

In conclusion, 20(S)-protopanaxatriol, a major metabolic product generated by intestinal bacteria after oral ingestion of PPT-type ginsenosides, has skin photoprotective properties that may occur through restoration of GSH levels, reduction of ROS production, and downregulation of MMP-2 and MMP-9. Taken together, these results suggested that 20(S)-PPT and other PPT-type ginsenosides must be investigated as natural antiphotoaging treatments. As natural products, they may have similar or enhanced activities with fewer side effects.

\section{Acknowledgements}

The authors would like to thank Ms. Hee Han for technical assistance. This study was supported by a grant of the Korean Health Technology R\&D Project, Ministry of Health \& Welfare, Republic of Korea (grant no. HN12C0060).

\section{References}

1. Bu QT, Zhang WY, Chen QC, Zhang CZ, Gong XJ, Liu WC, $\mathrm{Li} \mathrm{W}$ and Zheng YN: Anti-diabetic effect of ginsenoside Rb (3) in alloxan-induced diabetic mice. Med Chem 8: 934-941, 2012.

2. Chen J, Peng H, Ou-Yang X and He X: Research on the antitumor effect of ginsenoside Rg3 in B16 melanoma cells. Melanoma Res 18: 322-329, 2008.

3. Liu C, Zhang M, Hu MY, Guo HF, Li J, Yu YL, Jin S, Wang XT, Liu L and Liu XD: Increased glucagon-like peptide-1 secretion may be involved in antidiabetic effects of ginsenosides. J Endocrinol 217: 185-196, 2013.

4. Park EK, Choo MK, Han MJ and Kim DH: Ginsenoside Rh1 possesses antiallergic and anti-inflammatory activities. Int Arch Allergy Immunol 133: 113-120, 2004.

5. Seo YJ, Kwon MS, Choi HW, Jang JE, Lee JK, Sun Y, Jung JS, Park SH and Suh HW: Intracerebroventricular ginsenosides are antinociceptive in proinflammatory cytokine-induced pain behaviors of mice. Arch Pharm Res 31: 364-369, 2008.

6. Tao T, Chen F, Bo L, Xie Q, Yi W, Zou Y, Hu B, Li J and Deng X: Ginsenoside Rg1 protects mouse liver against ischemia-reperfusion injury through anti-inflammatory and anti-apoptosis properties. J Surg Res 191: 231-238, 2014

7. Wang W, Zhang X, Qin JJ, Voruganti S, Nag SA, Wang MH, Wang H and Zhang R: Natural product ginsenoside 25-OCH3-PPD inhibits breast cancer growth and metastasis through down-regulating MDM2. PLoS One 7: e41586, 2012.

8. Hasegawa H, Suzuki R, Nagaoka T, Tezuka Y, Kadota S and Saiki I: Prevention of growth and metastasis of murine melanoma through enhanced natural-killer cytotoxicity by fatty acid-conjugate of protopanaxatriol. Biol Pharm Bull 25: 861-866, 2002.

9. Oh GS, Pae HO, Choi BM, Seo EA, Kim DH, Shin MK, Kim JD, Kim JB and Chung HT: 20 (S)-Protopanaxatriol, one of ginsenoside metabolites, inhibits inducible nitric oxide synthase and cyclooxygenase- 2 expressions through inactivation of nuclear factor-kappaB in RAW 264.7 macrophages stimulated with lipopolysaccharide. Cancer Lett 205: 23-29, 2004.

10. Ahn HY, Hong SY, Kim JY and Kwon O: Panax ginseng extract rich in ginsenoside protopanaxatriol offers combinatorial effects in nitric oxide production via multiple signaling pathways. Springerplus 2: 96, 2013.

11. Han KL, Jung MH, Sohn JH and Hwang JK: Ginsenoside 20S-protopanaxatriol (PPT) activates peroxisome proliferator-activated receptor gamma (PPARgamma) in 3T3-L1 adipocytes. Biol Pharm Bull 29: 110-113, 2006. 
12. Hasegawa E, Nakagawa S, Miyate Y, Takahashi K, Ohta S, Tachikawa $E$ and Yamato S: Inhibitory effect of protopanaxatriol ginseng metabolite M4 on the production of corticosteroids in ACTH-stimulated bovine adrenal fasciculata cells. Life Sci 92 687-693, 2013.

13. Kwok HH, Ng WY, Yang MS, Mak NK, Wong RN and Yue PY: The ginsenoside protopanaxatriol protects endothelial cells from hydrogen peroxide-induced cell injury and cell death by modulating intracellular redox status. Free Radic Biol Med 48: $437-445,2010$

14. Han B, Meng Q, Li Q, Zhang J, Bi Y and Jiang N: Effect of 20(S)-protopanaxatriol and its epimeric derivatives on myocardial injury induced by isoproterenol. Arzneimittelforschung 61: 148-152, 2011.

15. Nordberg J and Arnér ES: Reactive oxygen species, antioxidants and the mammalian thioredoxin system. Free Radic Biol Med 31: $1287-1312,2001$.

16. Rabe JH, Mamelak AJ, McElgunn PJ, Morison WL and Sauder DN: Photoaging: Mechanisms and repair. J Am Acad Dermatol 55: 1-19, 2006.

17. Slominski A, Wortsman J and Tobin DJ: The cutaneous serotoninergic/melatoninergic system: Securing a place under the sun. FASEB J 19: 176-194, 2005.

18. Bradford MM: A rapid and sensitive method for the quantitation of microgram quantities of protein utilizing the principle of protein-dye binding. Anal Biochem 72: 248-254, 1976.

19. Royall JA and Ischiropoulos H: Evaluation of 2', 7'-dichlorofluorescin and dihydrorhodamine 123 as fluorescent probes for intracellular $\mathrm{H}_{2} \mathrm{O}_{2}$ in cultured endothelial cells. Arch Biochem Biophys 302: 348-355, 1993.

20. Sherman MP, Aeberhard EE, Wong VZ, Griscavage JM and Ignarro LJ: Pyrrolidine dithiocarbamate inhibits induction of nitric oxide synthase activity in rat alveolar macrophages. Biochem Biophys Res Commun 191: 1301-1308, 1993.

21. Freshney RI: Culture of animal cells: A manual of basic technique. 4th Edition. Wiley-Liss Press, New York, 1994.

22. Kleiner DE and Stetler-Stevenson WG: Quantitative zymography: Detection of picogram quantities of gelatinases. Anal Biochem 218: 325-329, 1994.

23. Nakagawa K, Saijo N, Tsuchida S, Sakai M, Tsunokawa Y, Yokota J, Muramatsu M, Sato K, Terada M and Tew KD: Glutathione-S-transferase pi as a determinant of drug resistance in transfectant cell lines. J Biol Chem 265: 4296-4301, 1990.

24. Quan T, Qin Z, Xia W, Shao Y, Voorhees JJ and Fisher GJ Matrix-degrading metalloproteinases in photoaging. J Investig Dermatol Symp Proc 14: 20-24, 2009.

25. Jenkins G: Molecular mechanisms of skin ageing. Mech Ageing Develop 123: 801-810, 2002.

26. Rittié L and Fisher GJ: UV-light-induced signal cascades and skin aging. Ageing Res Rev 1: 705-720, 2002.

27. Fisher GJ, Wang ZQ, Datta SC, Varani J, Kang S and Voorhees JJ: Pathophysiology of premature skin aging induced by ultraviolet light. N Engl J Med 337: 1419-1428, 1997.

28. Lee YM, Kang SM, Lee SR, Kong KH, Lee JY, Kim EJ and Chung JH: Inhibitory effects of TRPV1 blocker on UV-induced responses in the hairless mice. Arch Dermatol Res 303: 727-736, 2011.

29. Lee YM, Kim YK, Kim KH, Park SJ, Kim SJ and Chung JH: A novel role for the TRPV1 channel in UV-induced matrix metalloproteinase (MMP)-1 expression in HaCaT cells. J Cell Physiol 219: 766-775, 2009.
30. Choi HK, Kim DH, Kim JW, Ngadiran S, Sarmidi MR and Park CS: Labisia pumila extract protects skin cells from photoaging caused by UVB irradiation. J Biosci Bioeng 109: 291-296, 2010.

31. Kim J, Lee CW, Kim EK, Lee SJ, Park NH, Kim HS, Kim HK, Char K, Jang YP and Kim JW: Inhibition effect of gynura procumbens extract on UV-B-induced matrix-metalloproteinase expression in human dermal fibroblasts. J Ethnopharmacol 137: 427-433, 2011.

32. Krolikiewicz-Renimel I, Michel T, Destandau E, Reddy M, André P, Elfakir C and Pichon C: Protective effect of a Butea monosperma (Lam.) Taub. flowers extract against skin inflammation: Antioxidant, anti-inflammatory and matrix metalloproteinases inhibitory activities. J Ethnopharmacol 148: 537-543, 2013.

33. Piao MJ, Hyun YJ, Cho SJ, Kang HK, Yoo ES, Koh YS, Lee NH, Ko MH and Hyun JW: An ethanol extract derived from bonnemaisonia hamifera scavenges ultraviolet B (UVB) radiation-induced reactive oxygen species and attenuates UVB-induced cell damage in human keratinocytes. Mar Drugs 10: 2826-2845, 2012

34. Mu Y,LvS,Ren X,Jin G,Liu J,YanG,LiW,Shen J andLuo G: UV-B induced keratinocyte apoptosis is blocked by 2-selenium-bridged beta-cyclodextrin, a GPX mimic. J Photochem Photobiol B 69: $7-12,2003$.

35. Zhu M and Bowden GT: Molecular mechanism (s) for UV-B irradiation-induced glutathione depletion in cultured human keratinocytes. Photochem Photobiol 80: 191-196, 2004.

36. Meloni M and Nicolay JF: Dynamic monitoring of glutathione redox status in UV-B irradiated reconstituted epidermis: Effect of antioxidant activity on skin homeostasis. Toxicol In Vitro 17: 609-613, 2003

37. Varma SD, Hegde KR and Kovtun S: UV-B-induced damage to the lens in vitro: Prevention by caffeine. J Ocul Pharmacol Ther 24: 439-444, 2008.

38. Misra RB, Babu GS, Ray RS and Hans RK: Tubifex: A sensitive model for UV-B-induced phototoxicity. Ecotoxicol Environ Saf 52: 288-295, 2002

39. Jiang GZ and Li JC: Protective effects of ginsenoside Rg1 against colistin sulfate-induced neurotoxicity in PC12 cells. Cell Mol Neurobiol 34: 167-172, 2014

40. Ma J, Liu J, Wang Q, Yu H, Chen Y and Xiang L: The beneficial effect of ginsenoside Rg1 on schwann cells subjected to hydrogen peroxide induced oxidative injury. Int J Biol Sci 9: 624-636, 2013

41. Huang Q, Gao B, Jie Q, Wei BY, Fan J, Zhang HY, Zhang JK, Li XJ, Shi J, Luo ZJ, et al: Ginsenoside-Rb2 displays anti-osteoporosis effects through reducing oxidative damage and bone-resorbing cytokines during osteogenesis. Bone 66: 306-314, 2014

42. Liu YW, Zhu X, Li W, Lu Q, Wang JY, Wei YQ and Yin XX: Ginsenoside Re attenuates diabetes-associated cognitive deficits in rats. Pharmacol Biochem Behav 101: 93-98, 2012.

43. Ye R, Li N, Han J, Kong X, Cao R, Rao Z and Zhao G: Neuroprotective effects of ginsenoside Rd against oxygen-glucose deprivation in cultured hippocampal neurons. Neurosci Res 64: 306-310, 2009

44. Kim ND, Pokharel YR and Kang KW: Ginsenoside Rd enhances glutathione levels in H4IIE cells via NF-kappaB-dependent gamma-glutamylcysteine ligase induction. Pharmazie 62: 933-936, 2007. 